\section{L'Actualité économique}

L'ACTUALITÉ ÉCONOMIQUE

\section{Les défis d'une nouvelle théorie de la croissance}

\section{Philippe Aghion}

Volume 78, numéro 4, décembre 2002

URI : https://id.erudit.org/iderudit/007261ar

DOI : https://doi.org/10.7202/007261ar

Aller au sommaire du numéro

Éditeur(s)

HEC Montréal

ISSN

0001-771X (imprimé)

1710-3991 (numérique)

Découvrir la revue

Citer cet article

Aghion, P. (2002). Les défis d'une nouvelle théorie de la croissance. L'Actualité économique, 78(4), 459-486. https://doi.org/10.7202/007261ar
Ce document est protégé par la loi sur le droit d'auteur. L'utilisation des services d'Érudit (y compris la reproduction) est assujettie à sa politique d'utilisation que vous pouvez consulter en ligne.

https://apropos.erudit.org/fr/usagers/politique-dutilisation/ 


\title{
LES DÉFIS D’UNE NOUVELLE THÉORIE DE LA CROISSANCE
}

\author{
Philippe AGHION \\ Université Harvard \\ et University College London
}

\section{INTRODUCTION : LES TROIS GRANDS DÉFIS À RELEVER POUR UNE NOUVELLE THÉORIE DE LA CROISSANCE}

Dans son manuel de macroéconomie, publié en 1982, M. Edmond Malinvaud énonce plusieurs objectifs que, selon lui, toute bonne théorie de la croissance économique doit atteindre, notamment :

(i) expliquer les différents profils de croissance à long terme d'un pays à l'autre ou d'une région à l'autre, et formuler des relations vérifiables et stables entre la croissance à long terme et toute une gamme de facteurs économiques sous-jacents;

(ii) rendre compte des évolutions historiques à long terme (en particulier, la convergence ou la divergence des divers pays) et ainsi réconcilier la temporalité abstraite des modèles dynamiques avec le déroulement des changements économiques réels, analysés par les historiens;

(iii) éclairer d'un même souffle les transformations institutionnelles et les changements technologiques.

Cependant, comme Malinvaud a dû le reconnaître en 1982 lors de la parution de son manuel, les théories de la croissance de l'époque ne pouvaient prétendre atteindre aucun de ces objectifs, véritables défis légués aux générations suivantes de théoriciens. Dans cette conférence, nous soutiendrons que l'apparition, au cours des 12 dernières années, d'une nouvelle vague de modèles de croissance - dans lesquels la croissance est induite, non seulement par l'accumulation du capital et l'épargne, mais, plus fondamentalement, par les activités des entrepreneurs ou les innovations, lesquelles sont elles-mêmes stimulées ou facilitées par diverses caractéristiques de l'environnement institutionnel - a radicalement changé la situation, en mettant à notre portée la solution des 3 défis précités.

Cette conférence est structurée comme suit : la première section démontre brièvement que les modèles de croissance basés sur l'accumulation du capital ne 
peuvent pas expliquer, en même temps, la croissance à long terme et la convergence de divers pays, sans compter qu'ils sont pratiquement muets au sujet des institutions; la deuxième section présente le nouveau paradigme de la croissance et soutient que celui-ci permet pratiquement de relever les deux premiers défis; la troisième section montre, avant de conclure, que le nouveau paradigme de la croissance peut facilement être développé de façon à rendre endogènes les transformations institutionnelles et à les relier aux changements technologiques endogènes, répondant ainsi au troisième défi.

\section{LES LIMITES DES MODÈLES DE CROISSANCE BASÉS SUR L'ACCUMULATION DU CAPITAL}

\subsection{Le modèle néoclassique}

L'idée que la création de connaissances est critique pour la croissance économique à long terme est certainement la proposition la plus importante qui ressort de la théorie néoclassique de Solow (1956) et Swan (1956). Plus précisément, considérons une économie fermée dans laquelle la production finale $Y$ est assurée à chaque période en employant le capital disponible $K$, selon la technologie de production :

$$
Y=F(K, A L)
$$

où : (i) $A$ un est un paramètre de productivité qui mesure l'état actuel des connaissances; (ii) $L$ correspond à l'importance courante de la main-d'oeuvre; (iii) la technologie de production $F$ est sujette à des rendements décroissants par rapport à l'accumulation du capital, c'est-à-dire que la productivité marginale du capital $F_{K}$ baisse au fur et à mesure que le capital augmente.

Le capital s'accumule en conformité avec l'équation :

$$
\frac{d k}{d t}=s Y-\delta K
$$

où $s$ est le taux d'épargne - considéré constant dans le modèle de Solow-Swan et $\delta$ dénote le taux de dépréciation du capital.

En l'absence de croissance démographique (c'est-à-dire si $L$ reste constant) et de progrès technique (c'est-à-dire si $A$ est aussi constant), une telle économie ne peut pas croître indéfiniment à un taux positif. En effet, vu la diminution des rendements du capital, le revenu national $Y$ ne s'accroît pas aussi rapidement que le stock de capital, ce qui signifie que l'épargne $s Y$ ne peut pas augmenter aussi rapidement que la dépréciation. À un moment donné, la dépréciation rattrape l'épargne et, à ce point, le stock de capital stoppe sa progression et l'économie cesse de croître. Avec un accroissement démographique et une technologie de production $\mathrm{F}$ dont les rendements en fonction de $K$ et $L$ seraient constants, un raisonnement similaire pourrait s'appliquer à la production par personne $y=\frac{Y}{L}$, qui 
serait alors une fonction concave du capital par personne $k=\frac{K}{L}$; nous obtenons alors la proposition selon laquelle, en absence de création de connaissances (exogène), c'est-à-dire d'une croissance exogène de $A$, il ne peut y avoir aucune progression à long terme du revenu par personne quand la production finale enregistre des rendements décroissants par rapport à l'accumulation du capital. Autrement dit, le modèle néoclassique ne peut pas atteindre le premier objectif de Malinvaud, puisqu'il ne peut pas expliquer la hausse de la productivité à long terme.

Et qu'en est-il du deuxième objectif? De ce côté, les perspectives semblent un peu meilleures, du fait que le modèle néoclassique peut rendre compte du phénomène observable de convergence « conditionnelle », autrement dit du fait que parmi des pays ou les régions qui sont initialement suffisamment semblables, ceux dont le niveau de production ou de capital est le plus bas croissent plus rapidement que les pays qui sont plus proches de leur état stationnaire. Par exemple, si

$$
Y=K^{\alpha} L^{1-\alpha}
$$

alors, en divisant les deux membres de l'équation (AC) par $K$, on obtient directement :

$$
\frac{K}{K}=s\left(\frac{L}{K}\right)^{1-a}-\delta
$$

qui est évidemment une fonction décroissante de $K$. Donc, plus le capital s'est déjà accumulé dans un pays, plus le taux de croissance de ce pays est faible. Notez que la convergence entre les pays découle entièrement de l'hypothèse des rendements décroissants de l'accumulation du capital. Dans la section 2.2 ci-après, nous développerons une autre théorie de la convergence, basée sur la diffusion des connaissances d'un pays à l'autre.

\subsection{Le modèle AK}

La première variante du modèle de croissance endogène, appelé théorie $\mathrm{AK}$, a été présentée par Frankel (1962); les célèbres articles de Romer (1986) et de Lucas (1988) ont ensuite donné la formulation moderne de cette contribution, longtemps négligée. Essentiellement, le modèle AK traite la connaissance comme un type particulier de capital : la création de connaissances résulte directement de l'accumulation du capital par les différentes entreprises, l'idée de base étant que l'accumulation du capital contribue à la création collective de nouvelles connaissances technologiques et organisationnelles, par apprentissage sur le tas et imitation. Cette création de connaissances compensera de manière permanente la diminution de la productivité marginale du capital et permettra ainsi à l'économie de maintenir un taux de croissance positif à long terme, sous réserve des hypothèses appropriées concernant les externalités de l'apprentissage. 
D’abord présenté par Frankel (1962) pour réconcilier l'hypothèse des rendements décroissants de l'accumulation individuelle du capital avec la possibilité d'une croissance positive à long terme comme dans le modèle de Harrod-Domar, le modèle AK suppose une économie concurrentielle avec $N$ entreprises. Chaque entreprise $j(1 \leq j \leq N)$ produit son output final selon la fonction de production Cobb-Douglas :

$$
Y_{j}=A K_{j}^{\alpha} L_{j}^{1-\alpha}
$$

où : (i) $\alpha$ est strictement inférieur à 1 , de telle sorte que les rendements sont décroissants par rapport à l'accumulation individuelle du capital; (ii) $A$ est un paramètre de productivité, qui reflète l'état actuel des connaissances; tandis que l'évolution dynamique de $A$, c'est-à-dire la création des connaissances, qui est considérée comme donnée dans le modèle néoclassique discuté ci-dessus, est rendue endogène par le modèle $\mathrm{AK}$, car elle devient le résultat collectif de l'accumulation du capital par toutes les entreprises au sein de l'économie. Plus formellement, le modèle suppose :

$$
A=A_{0}\left(\frac{1}{N} \sum_{j} K_{j}\right)^{\eta}
$$

où $\eta$ quantifie les externalités dans l'apprentissage sur le tas par l'entreprise.

Pour simplifier, posons $L_{j} \equiv 1$ pour tous les $j$; alors, dans une situation d'équilibre symétrique, où $K_{j}=\frac{K}{N}$ pour tous les $j$, le revenu agrégé par personne $Y$ sera donné par l'équation :

$$
Y=A_{0} N^{1-\alpha-\eta} K^{\alpha+\eta}
$$

Cette équation et celle de l'accumulation (AC), qui est toujours valable ici si nous faisons l'hypothèse d'un taux d'épargne constant, détermineront la totalité du sentier de croissance de l'économie. Nous nous intéresserons particulièrement au cas limite où $\alpha+\eta=1$. Seulement en pareil cas, le taux de croissance à long terme $g$ sera positif et fini, étant égal à :

$$
g=s A_{0}-\delta,
$$

ce qui revient au taux de croissance du modèle de Harrod-Domar.

Une prédiction malheureuse du modèle $\mathrm{AK}$ de la croissance endogène, dans lequel la connaissance n'est considérée que comme du capital, est qu'une croissance positive à long terme n'est tout simplement pas compatible avec une possible convergence entre divers pays. Considérons en effet deux pays ou régions, chacun d'entre eux régi par le même type d'équations dynamiques que ci-dessus. Soit que ces deux pays (ou régions) partagent les mêmes caractéristiques fondamentales (en termes de taux d'épargne, de taux de dépréciation, de technologie de production, ...), alors, à partir du début, ces deux économies croîtront au même 
rythme $g=s A_{0}-\delta$; ou bien, ces pays auront des caractéristiques différentes, ou seront peut-être soumis à des chocs stochastiques, auquel cas leurs sentiers de croissance divergeront avec le temps. Le modèle néoclassique implique au contraire que, toutes choses étant égales par ailleurs, un pays riche qui a accumulé un stock de capital considérable doit croître plus lentement qu'un pays plus pauvre, possédant les mêmes paramètres économiques, mais dont le stock de capital est moindre. En fait, il existe des preuves très nettes d'une tendance à la convergence dans le revenu par personne, non seulement dans des régions aux conditions initiales différentes mais aux caractéristiques économiques semblables, comme certains États aux États-Unis, mais également entre des pays industrialisés et des économies de marché émergentes, en particulier dans le Sud-Est asiatique (voir Barro et Sala-i-Martín, 1995). Ces observations sur l'évolution des écarts de revenus d'un pays à l'autre, ont, à leur tour, servi de point d'appui pour critiquer l'ensemble de la théorie de la croissance endogène. Mankiw, Romer et Weil (1992) - que nous désignerons dorénavant par MRW - ont mené cette attaque de front, en soutenant que le modèle néoclassique de la croissance avec progrès technique exogène et rendements décroissants du capital (voir la sous-section 1.1 ci-dessus) peut expliquer la plus grande partie des variations dans la production par personne d'un pays à l'autre.

Si elle semble vouloir satisfaire, jusqu'à un certain point, le premier objectif (à savoir, expliquer la croissance à long terme), l'approche AK ne peut pas prétendre atteindre le deuxième objectif (en particulier, expliquer la convergence ou la divergence d'un pays à l'autre). En fait, le modèle AK ne satisfait pas tellement bien le premier objectif non plus. Par exemple, il ne peut pas rendre compte de la possibilité de maintenir une croissance positive optimale dans une économie où l'accumulation du capital exige l'utilisation d'une ressource non renouvelable. En effet, la seule façon de contrebalancer l'épuisement de ressources naturelles et de maintenir une croissance à long terme, consiste à faire intervenir le progrès technique. Cependant, dans la mesure où l'accumulation du capital reste le seul moteur des nouvelles connaissances dans ce modèle, l'accélération du progrès technique entraînerait un épuisement accru de la ressource naturelle, ce qui ne peut qu'assombrir les perspectives de croissance à long terme, autrement dit, qu'aggraver le problème que le progrès technique devait alléger! Plus fondamentalement, en privilégiant le rôle de l'offre d'épargne globale dans l'apparition de la croissance, le modèle AK néglige la demande et, plus spécifiquement, le rôle des entrepreneurs, des institutions et des politiques économiques pouvant rehausser la productivité en jouant sur les incitations des entrepreneurs à l'innovation ${ }^{1}$.

1. Cet accent excessif sur l'épargne occasionne des prédictions étranges, par exemple qu'une volatilité macroéconomique accrue devrait stimuler la croissance, étant donné qu'elle encouragerait à économiser davantage pour des motifs de précaution (voir Jones-Manuelli-Stacchetti, 1999). 


\section{UNE THÉORIE DE LA CROISSANCE À LA HAUTEUR DES DEUX PREMIERS DÉFIS}

La deuxième variante de la théorie de la croissance endogène est l'approche de Schumpeter ${ }^{2}$, qui repose sur les idées suivantes : (i) la principale source du progrès technologique est l'innovation; (ii) les innovations, qui mènent à l'introduction de nouveaux procédés de production, de nouveaux produits, de nouvelles méthodes de gestion, de même qu'à une nouvelle organisation des activités de production, sont le fruit d'entreprises, d'entrepreneurs et de chercheurs qui sont motivés par leurs propres intérêts et qui s'attendent à se voir récompensés avec des rentes (de monopole) si leurs innovations connaissent du succès ${ }^{3}$; (iii) en général, ces rentes monopolistiques finissent par disparaître, au fur et à mesure que les processus ou produits deviennent désuets quand arrivent d'autres innovations qui rivalisent avec les technologies en place et les chassent du marché; c'est la notion schumpetérienne de « destruction créatrice ».

À la différence de ses prédécesseurs de type AK, le modèle de Schumpeter distingue, d'une part, la $\mathrm{R}$ et $\mathrm{D}$ et les connaissances et, d'autre part, les investissements dans le capital physique ou humain. Dans la section 2.1, nous donnerons une représentation schématique de ce cadre conceptuel. La section 2.2 exposera le modèle de Schumpeter de base et l'emploiera pour analyser les déterminants institutionnels de la croissance (à long terme). Prises dans leur ensemble, les sections 2.1 et 2.2 illustreront comment ce cadre conceptuel se situe par rapport au premier objectif énoncé par Malinvaud. La section 2.3 se concentrera sur le deuxième objectif, avec une analyse des types de convergence d'un pays à l'autre. En particulier, elle démontrera, premièrement que, contrairement au modèle $A K$, l'approche de Schumpeter peut admettre la convergence d'un pays à l'autre et, deuxièmement, qu'elle surpasse l'approche néoclassique en intégrant d'autres phénomènes, comme la convergence sélective (« club convergence »).

\subsection{Les institutions et la croissance à long terme}

\subsubsection{Aperçu de la théorie de la croissance de Schumpeter}

Trois grandes idées sont à la base de ce que nous appelons généralement, à l'heure actuelle, les nouvelles théories de la croissance :

2. Cette approche, qui se fonde sur Aghion-Howitt (1992), est développée en détails dans Aghion-Howitt (1998). Voir aussi Romer (1990) pour un modèle de croissance basé sur la R et D et qui n'utilise pas la notion de destruction créatrice, mise de l'avant par Schumpeter; et également Grossman-Helpman (1991), pour un modèle à échelle de qualités avec demande élastique unitaire, combinant Aghion-Howitt (1992) et Segerstrom, Anant et Dinopoulos (1990).

3. Naturellement, la création des connaissances dépend aussi des progrès de la science fondamentale, lesquels sont souvent plutôt le fruit de la curiosité que de l'appât du gain. Pourtant, beaucoup des recherches qui ont mené à des percées scientifiques ont été conduites par des entreprises privées et à but lucratif. 
- la croissance est principalement stimulée par le taux d'innovations technologiques, sous forme de nouveaux produits, de nouvelles méthodes et de nouvelles façons d'organiser les processus de production;

- la plupart des innovations découlent des activités des entrepreneurs ou des investissements - généralement, des investissements dans la R et D - et comportent des risques, liés à l'expérimentation et à l'apprentissage ${ }^{4}$;

- la motivation à investir dans l'innovation est elle-même influencée par l'environnement économique 5 .

Le graphique 1 peut nous aider à illustrer ce nouveau paradigme, qui, contrairement aux modèles de croissance précédents, n'est pas du tout basé sur l'accumulation du capital et l'épargne globale. L'axe horizontal montre la quantité de capital par unité d'efficacité du travail à l'état stationnaire, $k$, tandis que l'axe vertical indique le taux de croissance de la productivité à l'état stationnaire, $g^{6}$.

4. Un exemple pertinent est la « révolution verte » ou l'augmentation de la production de céréales résultant de la mise au point scientifique de nouvelles variétés de semences hybrides (blé, riz et maïs), qui ont fortement rehaussé les rendements agricoles dans nombre de pays en développement.

5. Par exemple, une probabilité élevée d'imitation, c'est-à-dire une piètre protection des droits de propriété, aura tendance à réduire les profits attendus d'un innovateur ayant du succès, et découragera ainsi l'investissement dans l'innovation. Un autre exemple nous est donné par les taux d'intérêt élevés qui résultent de la volatilité macroéconomique. De tels taux réduiront la valeur actualisée des rentes que toucherait un innovateur et décourageront donc les activités novatrices. Dans la dernière section, nous compléterons cette liste en expliquant clairement ce que nous considérons comme les principales embûches à l'esprit d'entreprise dans les régions pauvres.

6. Formellement :

$$
k=\frac{K}{A L}
$$

où $K$ est le stock de capital, $L$ correspond à la main-d'oeuvre employée dans la fabrication du produit final et $A$ dénote la productivité courante du travail. À l'état stationnaire, le taux de croissance de $A$ est

$$
g=\frac{A}{A}
$$




\section{GRAPHIQUE 1}

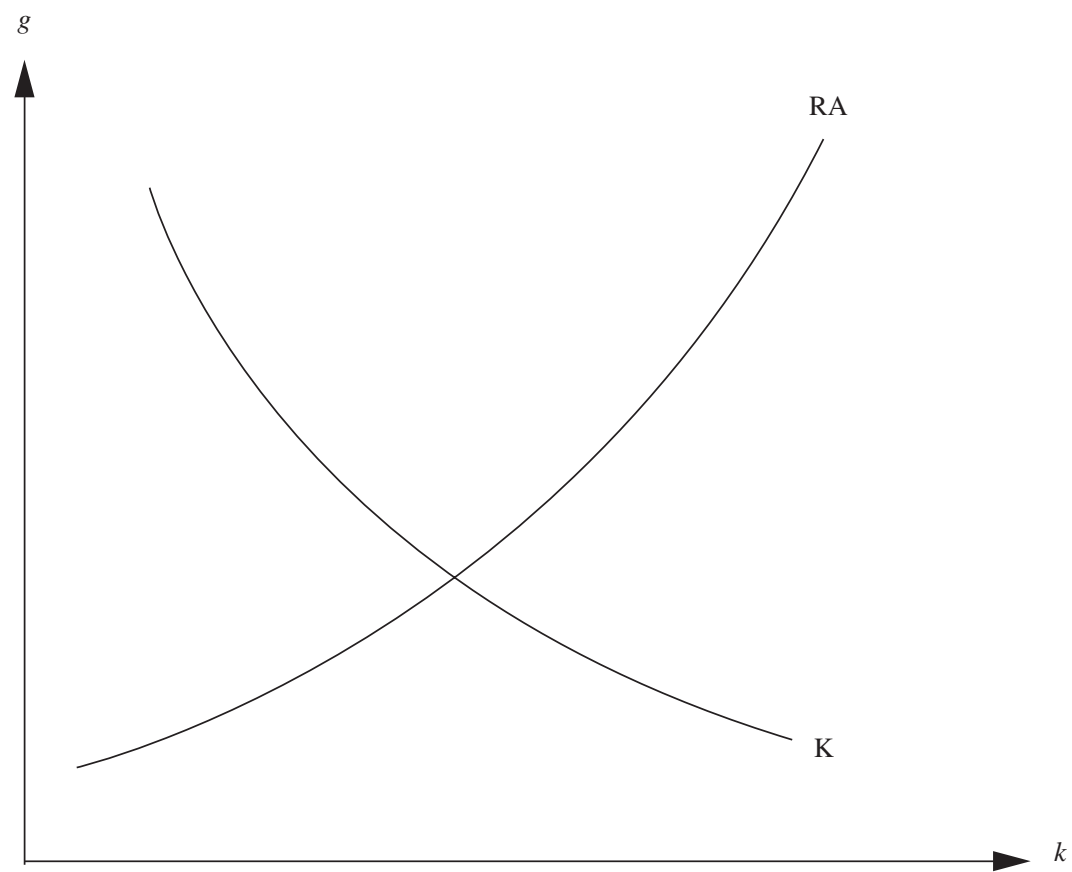

La courbe $\mathrm{K}$, dont la pente est négative, représente, la quantité de capital à l'état stationnaire par unité d'efficacité du travail en fonction du taux de croissance de la productivité. Par exemple, celle qui provient de l'équation d'accumulation du capital dans le modèle Solow ${ }^{7}$.

7. Nous savons que dans le modèle de Solow - ou dans l'équation d'Euler du modèle de Ramsey - le flux de production est donné par une équation de la forme

$$
Y=K^{\alpha}(A L)^{1-\alpha}
$$

où l'accumulation nette du capital provient de la différence entre l'épargne $s Y$ et la dépréciation $\delta K$, de telle sorte que

$$
K=s Y-\delta K,
$$

nous obtenons donc :

$$
k=s k^{\alpha}-(\delta+n+g) k
$$

où $\mathrm{n}$ désigne le taux de croissance de la population et $\mathrm{g}$, le taux de croissance de la productivité. Ainsi, à l'état stationnaire :

$$
k=\left(\frac{\delta+n+g}{s}\right)^{\frac{1}{\alpha-1}}
$$

ce qui correspond bien à une fonction décroissante de $g$. 
Plus le taux de croissance de la productivité est élevé, plus le capital par unité d'efficacité du travail se déprécie rapidement, abaissant par le fait même la quantité de capital par unité d'efficacité du travail à l'état stationnaire. La courbe à pente positive RA représente le taux de croissance de la productivité à l'état stationnaire en fonction de la quantité de capital par unité d'efficacité du travail à l'état stationnaire ${ }^{8}$.

Intuitivement, il est facile de comprendre que plus le stock de capital par personne, ajusté pour la productivité, est élevé, plus le revenu par personne l'est aussi et, donc, plus importante est la rente monopolistique qui vient récompenser l'innovation heureuse. Ainsi, plus grande est l'incitation à l'activité innovatrice. Et, en fin de compte, le taux de croissance de la productivité s'en trouve accru, puisque ce taux est tiré par le taux d'innovations.

À l'intersection des deux courbes K et RA, se trouve le taux d'équilibre de la croissance à long terme de la productivité, qui dépend non seulement du taux global d'épargne, mais encore d'un ensemble de politiques ou de variables institutionnelles, ce qui peut nous faire considérablement progresser vers l'atteinte du premier objectif énoncé par Malinvaud (1982). Plus spécifiquement, à l'instar des modèles de croissance précédents basés sur l'accumulation du capital, un accroissement du taux d'épargne décalerait la courbe $\mathrm{K}$ vers la droite. Cependant, un large éventail de politiques ou de particularités institutionnelles jouent ici un rôle crucial - par l'entremise de la courbe RA. Mentionnons, par exemple, la protection des droits de propriété intellectuelle, les subventions aux activités innovatrices, la concurrence sur le marché, la volatilité macroéconomique, l'offre de main-d'œuvre qualifiée ou l'efficacité de la recherche, etc. Tous ces paramètres ont des répercussions sur la croissance, par l'entremise de leurs effets sur la courbe RA. Généralement, une meilleure protection des droits de propriété intellectuelle, des subventions accrues aux activités innovatrices, une plus grande efficacité de la recherche ou une abondance de travailleurs qualifiés déplaceront la courbe RA vers le haut, stimulant ainsi la croissance de la productivité. Parallèlement, un accroissement de la volatilité macroéconomique ou un resserrement des limites de crédit déplacerait la courbe RA vers le bas, réduisant ainsi la croissance de la productivité.

\subsubsection{Le modèle de Schumpeter ${ }^{9}$}

Dans la présente sous-section, nous mettrons en équations le cadre conceptuel. Nous considérons tout d'abord une économie fermée qui fabrique un seul produit

8. Formellement, cette courbe RR rend compte de deux relations : d'abord, une équation de croissance, selon laquelle le taux de croissance à l'état stationnaire est une fonction croissante du taux d'innovations et ainsi de l'investissement (à l'état stationnaire) dans des activités innovatrices; deuxièmement, une équation «d'arbitrage de la recherche », qui lie l'investissement innovateur à diverses caractéristiques de l'environnement économique, en particulier à la taille de l'économie telle que mesurée par le stock de capital par personne à l'état stationnaire, mais aussi au taux d'intérêt, à la productivité de la $\mathrm{R}$ et $\mathrm{D}$, à la probabilité d'imitation, à la concurrence sur le marché, etc.

9. Voir Aghion-Howitt (1998, chapitre 3). 
fini, lequel peut être voué aussi bien à la consommation qu'à la production à titre d'intrant intermédiaire. Ce produit fini est fabriqué en conformité avec la technologie de production :

$$
Y=L^{1-\alpha} \int_{0}^{1} A_{i} x_{i}^{\alpha} d i
$$

où $L$ est le flux de travail employé dans la fabrication du produit fini, $x_{i}$ correspond à la quantité d'intrant $i$ couramment utilisée pour fabriquer ce produit, et $A_{i}$ est un paramètre de productivité mesurant la qualité de la dernière version de l'intrant $i$ (pour simplifier, nous omettons l'indice de temps $t$ dans cette équation).

Les intrants intermédiaires sont tous produits en employant le capital selon la fonction de production :

$$
x_{i}=\frac{K_{i}}{A_{i}}
$$

où $K_{i}$ correspond l'entrée de capital dans le secteur $i$. La division par $A_{i}$ reflète le fait que les générations successives d'intrants intermédiaires sont fabriquées par des technologies de plus en plus capitalistiques.

La création des connaissances, c'est-à-dire les innovations technologiques, vise des intrants intermédiaires particuliers. Une innovation dans le secteur $i$ provoquera une amélioration de l'intrant intermédiaire $i$ et, en même temps, permettra à son promoteur de remplacer le monopoleur en place jusqu'à ce qu'une innovation ultérieure vienne le déloger ${ }^{10}$.

Le monopoleur en place dans chaque secteur intermédiaire $i$ fonctionne avec un barème de prix donné par la productivité marginale de l'input $i$, à savoir :

$$
p_{i}=A_{i} \alpha x_{i}^{\alpha-1} L^{1-\alpha}
$$

et une fonction de coûts linéaire, égale à :

$$
C\left(x_{i}\right)=(r+\delta-\beta) K_{i}=(r+\delta-\beta) A_{i} x_{i}
$$

où $r$ est le taux d'intérêt courant (de nouveau, pour simplifier la notation, nous omettons l'indice temporel $t$ ); $\delta$ correspond au taux fixe de dépréciation et $\beta$ désigne le taux auquel l'accumulation du capital est subventionnée. Ainsi, en normalisant l'offre globale de travail $L$ à 1 (pour simplifier), le monopoleur en place dans le secteur $i$ choisira $x_{i}$ de façon à maximiser :

10. Dans ce modèle, comme dans celui d'Aghion-Howitt (1992), aucune innovation n'est le fait des monopoleurs en place; cela découle directement : (i) du fait que les nouvelles connaissances deviennent immédiatement accessibles à des chercheurs autres que ceux des entreprises en place ; (ii) de l'effet Arrow (ou de remplacement), selon lequel l'accroissement bénéficiaire lié à l'innovation est moindre pour une entreprise en place que pour une nouvelle entreprise, puisque la première jouit déjà d'une rente de monopole positive; (iii) du fait que la technologie de recherche est linéaire. 


$$
\max \left\{A_{i} \alpha x_{i}^{\alpha-1} \cdot x_{i}-(r+\delta-\beta) A_{i} x_{i}\right\}=\pi_{i}
$$

On voit immédiatement que la solution $x$ à ce programme de maximisation est indépendante de $i$; c'est-à-dire qu'à l'équilibre, toutes les entreprises intermédiaires fourniront la même quantité de produits intermédiaires, ce qui implique que pour tout $i$ :

$$
\frac{K_{i}}{a_{i}} \equiv x \equiv \frac{K}{A}=k
$$

où $K=\int K_{i} d i$ est la demande globale de capital, qui, à l'équilibre, est égale à l'offre globale de capital; $A=\int A_{i} d i$ dénote le paramètre de productivité moyenne dans tous les secteurs et, par conséquent, $k=\frac{K}{A}$ correspond au stock de capital par travailleur productif. La condition de premier ordre pour le programme de maximisation peut alors être simplement réécrite comme suit :

$$
\alpha^{2} k^{\alpha-1}=r+\delta-\beta
$$

Nous appellerons cette première condition d'équilibre l'équation de capital et nous la noterons $(\mathrm{K})$. La deuxième condition sera analogue à l'équation d'arbitrage de la recherche dans Aghion-Howitt (1992, 1998), que nous déduirons de la façon que nous décrirons maintenant.

Comme dans Aghion-Howitt (1992), les innovations résultent des investissements dans la $\mathrm{R}$ et $\mathrm{D}$, mais ici nous supposons qu'au lieu d'employer uniquement le travail comme facteur de production, les secteurs de $\mathrm{R}$ et $\mathrm{D}$ utilisent des biens finals, ou, d'une façon équivalente, absorbent du travail et du capital selon la même technologie Cobb-Douglas que le secteur des produits finis. Une innovation dans le secteur $i$ à la date $t$ hissera le paramètre de productivité $A_{i}$ de ce secteur jusqu'à la productivité de pointe du moment $A^{\max }=\max _{j} A_{j}$. Implicitement, cela suppose que la technologie de pointe, une fois découverte, est automatiquement révélée et devient par conséquent immédiatement disponible pour tous les innovateurs potentiels. Ainsi, tandis que l'innovateur en place dispose d'un pouvoir de monopole sur l'utilisation de son innovation, la connaissance incarnée dans cette innovation est accessible à tous les producteurs engagés dans des activités de $\mathrm{R}$ et $\mathrm{D}$, et cherchant eux-mêmes à innover.

On suppose que les innovations dans n'importe quel secteur intermédiaire suivent un processus de type Poisson, avec un taux d'occurrence de :

$\lambda n$

où $\lambda$ est un paramètre qui mesure la productivité de la $\mathrm{R}$ et $\mathrm{D} ; n$ correspond à la quantité de produits finis consacrés à la $\mathrm{R}$ et $\mathrm{D}$, ajustée pour la productivité, ou, plus précisément, les dépenses de $\mathrm{R}$ et $\mathrm{D}$ par bien intermédiaire, divisées par le 
niveau de la productivité de pointe $A^{\max 11}$; nous divisons par $A^{\max }$ pour refléter le fait que, au fur et à mesure que la technologie progresse, le coût des ressources utilisées pour ces avancées augmente proportionnellement ${ }^{12}$.

La condition d'arbitrage de la recherche déterminant le niveau d'équilibre de la $\mathrm{R}$ et $\mathrm{D}$ stipule simplement que le coût marginal net de la $\mathrm{R}$ et $\mathrm{D}$ - à savoir, $1-\psi$, où $\psi$ est le taux selon lequel la $\mathrm{R}$ et $\mathrm{D}$ est subventionnée (ou imposée, si $\psi<0$ ) - est égal à la valeur prévue - après redressement pour tenir compte de la productivité - d'une unité de production finale investie dans la $\mathrm{R}$ et $\mathrm{D}$; cette valeur prévue est égale à $\frac{\lambda}{A^{\max }}$ fois la valeur de l'innovation dans n'importe quel secteur intermédiaire, qui à son tour correspond à :

$$
V=\frac{\pi}{r+\lambda n}
$$

où $\pi=\max _{x_{i}}\left\{A_{i} \alpha x_{i}^{\alpha-1} \cdot x_{i}-(r+\delta-\beta) A_{i} x_{i}\right\}=A^{\max } \cdot \pi(k)=A^{\max } \alpha(1-\alpha) k^{\alpha} \quad$ (ici, nous supposons implicitement que l'innovation pousse la productivité $A_{i}$ dans le secteur $i$ jusqu'au niveau de pointe actuel $A^{\text {max }}$ ). Le dénominateur de (RA) correspond au taux d'actualisation des innovations en place; il est égal au taux d'intérêt plus le taux de destruction créatrice $\lambda n$, c'est-à-dire la probabilité instantanée d'être déclassée par une nouvelle innovation arrivant dans le secteur. De là, nous obtenons une simple équation d'arbitrage de la recherche, que nous noterons encore (RA) :

$$
1-\psi=\lambda\left(\frac{\pi(k)}{r+\lambda n}\right) .
$$

Les équations (K) et (RA) déterminent conjointement l'équilibre stationnaire de la $\mathrm{R}$ et $\mathrm{D}$ en fonction des paramètres de l'économie. En particulier, le niveau d'équilibre de la $\mathrm{R}$ et $\mathrm{D}$ sera rehaussé par la faiblesse des taux d'intérêt, ou par une augmentation du taux des subventions de la $\mathrm{R}$ et $\mathrm{D}, \psi$, ou encore par un accroissement de la productivité de la $\mathrm{R}$ et $\mathrm{D}, \lambda$; d'autre part, l'équilibre sera abaissé par une hausse du coût du capital (par exemple, après une augmentation du taux de dépréciation $\delta$ ); enfin, il réagira positivement à toute législation sur les brevets, protégeant les innovateurs contre le risque d'imitation (si les innovations peuvent être imitées au taux de Poisson $p$, alors le dénominateur dans le membre de droite de $(\mathrm{R})$ deviendra $r+\lambda n+p)$.

Puis, pour passer de la $\mathrm{R}$ et $\mathrm{D}$ à la croissance, nous supposerons une diffusion des connaissances d'un secteur à l'autre, occasionnant une croissance de la productivité de pointe $A^{\max }$ à un taux proportionnel au flux des innovations dans l'économie, autrement dit :

11. Évidemment, $A^{\max }$ reste proportionnelle à la productivité moyenne à l'état stationnaire.

12. Cette hypothèse des « occasions décroissantes » est exposée et analysée en détails par Kortum (1997). 


$$
\frac{{ }^{\max }}{A^{\max }}=\lambda n \sigma=g
$$

où $\alpha>0$ mesure l'importance de la diffusion d'un secteur à l'autre. Alors, la statique comparative de l'équilibre de la $\mathrm{R}$ et $\mathrm{D}$, évoquée précédemment, se répercutera immédiatement sur le taux de croissance à l'équilibre $g$, qui, à l'état stationnaire, correspond également au taux de croissance de la productivité moyenne, c'est-à-dire : $\frac{\bullet A}{A}=g$, puisque la distribution des ratios de productivité $\frac{A_{i}}{A^{\max }}$ est alors stationnaire. Nous obtenons alors la courbe RA.

\subsection{La convergence des pays et la diffusion des connaissances ${ }^{13}$}

Avec leur accent sur les institutions, les nouvelles théories de la croissance peuvent, de toute évidence, faire plus que traiter de la croissance à long terme et des activités de $\mathrm{R}$ et $\mathrm{D}$ de pointe dans les économies de l'OCDE. En particulier, elles peuvent éclaircir pourquoi quelques régions, qui étaient initialement pauvres - comme en fait foi leur produit intérieur brut par personne - par exemple l'Asie, ont réussi à s'en sortir pour éventuellement atteindre des produits intérieurs bruts par personne comparables à ceux des pays industrialisés, alors que d'autres régions pauvres, par exemple l'Afrique, sont restées sous-développées.

L'explication de ce phénomène de « convergence sélective », suggérée par les nouvelles théories de la croissance, est simple: il s'agit essentiellement d'ajouter à ce cadre conceptuel une hypothèse de diffusion des connaissances, selon laquelle n'importe quel secteur dans les pays les moins développés peut parfaitement se mettre à l'heure des technologies de pointe à chaque fois qu'il « innove ». Dans un tel contexte, le terme "innovation » se réfère aussi à l'adaptation, au marché local ou à des conditions géographiques particulières, de technologies ou de produits tout d'abord inventés dans des pays plus avancés. L'hypothèse de la diffusion des connaissances implique quant à elle, que plus un pays tire de l'arrière, plus le bond moyen qu'il effectuera en innovant sera grand, et, par conséquent, plus son taux de croissance pour une intensité d'innovation donnée sera élevé. Ce qui distingue maintenant les pays qui convergent de ceux qui stagnent est, de nouveau, de nature institutionnelle : (i) les pays pauvres où, par exemple, les conditions macroéconomiques, le système juridique et le système d'éducation sont suffisamment favorables pour que les entrepreneurs puissent exercer leurs talents profiteront de la diffusion des connaissances et convergeront ainsi vers la technologie de pointe (frontière technologique); (ii) les pays pauvres où un marché du crédit bien structuré est essentiellement inexistant, le droit de propriété est

13. Cette section provient entièrement de Howitt (2000). 
difficile à faire respecter, l'instabilité économique et politique endémique, ou le niveau d'analphabétisme reste considérable dissuaderont les entrepreneurs d'exercer leurs activités. De tels pays ne peuvent que stagner et rester pauvres ${ }^{14}$.

Pour formaliser cela, considérons une économie mondiale composée de $m$ pays, désignés par les indices $j \in\{1,2, \ldots, m\}$. Chaque pays produit selon la technologie de production indiquée en (5). La principale particularité se trouve dans l'hypothèse de la diffusion technologique mondiale : c'est-à-dire qu'à n'importe quel moment, il existe un paramètre de technologie de pointe mondiale $A^{\text {max }}$ tel que :

$$
A^{\max }=\max \left\{A_{i j} ; i \in[0,1], 1 \leq j \leq m\right\}
$$

où $A_{i j}$ désigne le niveau de productivité courant dans le secteur $i$ du pays $j$; nous supposons alors qu'une innovation se produisant dans le secteur $i$ d'un pays aboutit à une nouvelle génération d'intrants intermédiaires $i$, dont la productivité rejoint le paramètre mondial de pointe $A^{\mathrm{max}}$.

Dans chaque pays, la technologie innovatrice est la même que dans le modèle à un seul pays de la sous-section 1.3; mais tous les pays novateurs croîtront à long terme au taux mondial :

$$
g=\frac{\bullet A^{\max }}{A^{\max }}=\sum_{1 \leq j \leq m} \sigma_{j} \lambda_{j} n_{j}
$$

où les $\sigma_{j}$ sont des coefficients de diffusion non négatifs, les $\lambda_{j}$ mesurent la productivité de $\mathrm{R}$ et $\mathrm{D}$ dans le pays $j$, et $n_{j}$ quantifie l'intensité de la $\mathrm{R}$ et $\mathrm{D}$ dans le pays $j$.

Supposons que $A$ désigne la productivité moyenne courante d'un pays particulier (nous omettons l'indice $j$ pour alléger la notation). Ce paramètre croîtra avec le temps, en raison des innovations provenant du pays lui-même, car chaque innovation pousse le secteur dans lequel elle se produit jusqu'au niveau de pointe courant $A^{\text {max }}$. Puisque les innovations ont la même probabilité d'occurrence dans tous les secteurs de l'économie nationale, la croissance de la productivité moyenne est décrite par l'équation différentielle :

$$
A=\lambda n\left(A^{\max }-A\right) .
$$

14. On pourrait développer ce cadre conceptuel, par exemple, en supposant que si le bond technologique s'accroît en fonction de l'écart par rapport à la technologie de pointe (à la faveur de la diffusion technologique), la fréquence des innovations dépend du ratio [écart par rapport à la technologie de pointe] / [bassin de main-d'œuvre qualifiée]. Ce cadre enrichi (voir Howitt-Mayer, 2002) pourrait expliquer, non seulement pourquoi quelques pays convergent tandis que d'autres stagnent, mais aussi pourquoi certains pays peuvent effectivement enregistrer des évolutions positives en terme de croissance, mais pourtant diverger sur le long terme. 
Plus spécifiquement, un pays dont le taux d'innovations $\lambda$ n est plus élevé sera en moyenne plus productif qu'un autre parce qu'une plus grande proportion de ses secteurs aura récemment innové et aura ainsi déplacé les paramètres de productivité jusqu'à rejoindre la frontière technologique courante. Maintenant, posons : $a \equiv \frac{A}{A^{\max }}$ pour dénoter la productivité intérieure moyenne du pays en regard de la productivité de pointe. En divisant les deux membres de l'équation différentielle précédente $\operatorname{par} A^{\max }$, et en tirant parti du fait que $g=\frac{\bullet A^{\max }}{A^{\max }}$, nous obtenons l'équation différentielle suivante pour $a$ :

$$
a=\lambda n(1-a)-a g
$$

Cette équation décrit le mécanisme par lequel les transferts de connaissances produisent une convergence vers les taux de croissance mondiaux. Une augmentation de la $\mathrm{R}$ et $\mathrm{D}$ stimulera temporairement la croissance de la productivité, mais à mesure que l'écart $(1-a)$ entre la productivité moyenne du pays et celle de pointe dans le monde se rétrécit, les innovations relèveront la productivité de moins en moins, ce qui ralentira la progression de la productivité moyenne du pays. Cela provoquera la convergence vers le même taux de croissance $g$... de tous les pays qui entreprennent de la $\mathrm{R}$ et $\mathrm{D}$.

Mais ce ne sont pas tous les pays qui entreprendront de la $\mathrm{R}$ et $\mathrm{D}$ à l'équilibre : lorsque nous avons déduit l'équation d'arbitrage de la recherche, nous avons implicitement limité l'analyse au cas où l'intensité d'équilibre de la recherche $n$ est strictement positive. Plus généralement, la condition d'arbitrage de la recherche s'exprime comme suit :

$$
1-\psi \geq \lambda \frac{\pi(k)}{r+\lambda n} ; n \geq 0, \text { avec au moins une égalité. }
$$

Plus spécifiquement, un pays $j$ dans lequel la productivité de la $\mathrm{R}$ et $\mathrm{D} \lambda_{j}$ est très basse ou les subventions de $\mathrm{R}$ et $\mathrm{D}, \psi$, sont faibles ou les rentes liées à l'innovation sont inappropriées (c'est-à-dire pour un $k$ donné, $\pi(k)$ est médiocre) ou le taux d'intérêt $r$ est élevé, ce pays, dis-je, restera piégé dans une situation d'absence d'innovation ou de croissance, avec $n=0$ à l'état stationnaire; d'autre part, les pays dont la productivité de $\mathrm{R}$ et $\mathrm{D}$ est plus considérable, avec des rentes plus appropriées et de faibles taux d'intérêt, entreprendront de la R et D et convergeront ainsi vers le taux de croissance commun $g$. Ainsi, seulement les membres de ce « club» sélect convergeront, tandis que les pays les plus pauvres seront laissés pour compte en l'absence d'aide publique ou étrangère.

Ainsi, contrairement à son prédécesseur le modèle AK, le modèle de croissance de Schumpeter peut expliquer la convergence ou la divergence d'un pays à l'autre, en même temps que la croissance à long terme; autrement dit, il peut atteindre les deux premiers objectifs formulés par Malinvaud. 


\section{EXPLICATION DES TRANSFORMATIONS INSTITUTIONNELLES}

Ainsi, contrairement aux modèles de croissance antérieurs basés sur l'accumulation du capital, les nouvelles théories de la croissance peuvent donner une explication à la fois des écarts dans les taux de croissance à long terme et des évolutions convergentes ou non. Dans la mesure où ils donnent beaucoup d'importance aux institutions, ces théories ne s'écartent pas tellement des préoccupations traditionnelles des économistes en développement. Et pourtant, on pourrait soutenir que ces nouvelles théories de la croissance restent peu utiles pour formuler des politiques de développement : elles semblent préconiser une politique identique pour tous les pays, quel que soit leur niveau de développement, et notamment leur stabilité macroéconomique, leur respect des droits de propriété, l'excellence de leur système d'éducation et également le développement de leur système financier et sa capacité à encourager l'épargne et la prise de risques. Plus fondamentalement, les nouvelles théories de la croissance analysées ci-dessus semblent incapables d'expliquer l'évolution des institutions, par exemple le fait, noté par Gerschenkron (1962), que les économies les moins développées semblent caractérisées par une concurrence moins vive, davantage d'interventions étatiques et de financement bancaire, par un faible roulement des gestionnaires et davantage d'intégration verticale des entreprises, alors que celles qui sont à la fine pointe de la technologie ont tendance à compter davantage sur le marché financier et l'impartition et semblent caractérisées par une concurrence plus vive et par un plus fort roulement des gestionnaires. Dans la présente section, nous soutiendrons qu'on peut facilement étendre la nouvelle théorie de la croissance pour prendre en compte de tels phénomènes et, plus généralement, relever le troisième défi mentionné dans l'introduction, à savoir que l'explication des transformations institutionnelles doit également rendre compte du changement technologique. La section est organisée comme suit : dans la sous-section 3.1, nous aborderons la question des institutions favorisant la croissance et ses implications pour une politique de développement; dans la sous-section 3.2, nous présenterons un exemple analytique simple (tiré directement d'Acemoglu-Aghion-Zilibotti (2002b) pour illustrer comment la nouvelle théorie de la croissance peut servir à expliquer certains aspects des transformations institutionnelles, et nous nous concentrerons alors sur l'intégration verticale.

\subsection{La notion d'institutions appropriées ${ }^{15}$}

Au cours des deux dernières décennies, nous avons assisté à un vif débat concernant les institutions et les politiques gouvernementales les plus favorables au développement économique et au rattrapage technologique. Chez les stratèges et les conseillers en poste à la Banque mondiale et dans d'autres institutions financières internationales, l'enthousiasme initial pour les stratégies reposant sur l'investissement, comme celles appliquées en Corée et au Japon, a pratiquement

15. Cette sous-section s'inspire fortement d'Acemoglu-Aghion-Zilibotti (2002a). 
disparu. Depuis le début des années quatre-vingt, ces stratégies ont été balayées par une profonde croyance dans des stratégies de marché et de libéralisme économique, du type de celles qui ont été appliquées à Hong Kong ou au Royaume-Uni au XIX ${ }^{\mathrm{e}}$ siècle. (Cette croyance si répandue est aussi - et très souvent - appelée le Consensus de Washington). Le débat sur la stratégie de développement la plus appropriée a atteint les universités, avec une ligne de démarcation très nette entre, d'une part, les positions d'universitaires comme Joseph Stiglitz (1995) ou Ricardo Hausmann et Dani Rodrik (2002) et, d'autre part, celles d'Andrei Shleifer et Robert Vishny (1999). Les premiers préconisent fortement l'intervention du gouvernement et les subventions dans les pays les moins développés, dans lesquels les externalités et les lacunes du marché sont légion, alors que les derniers soutiennent que les pays en retard sont non seulement affectés par les lacunes du marché, mais, aussi et peut-être davantage, par des déficiences gouvernementales et de nombreux détournements des politiques au profit d'intérêts privés, ce qui augmente les dangers d'une politique interventionniste.

Ce débat a été alimenté par l'expérience de divers pays pendant les 50 dernières années. Plus précisément, par les résultats contrastés des politiques interventionnistes d'un pays à l'autre jusqu'à la fin des années soixante-dix, de même que dans certains pays avant, pendant et après les années quatre-vingt. Ainsi, tandis que des stratégies reposant sur l'investissement ont stimulé la croissance dans des pays comme la Corée du Sud, le Japon, le Pérou et le Mexique entre les années cinquante et les années soixante-dix, des politiques semblables ont souvent échoué, notamment en Afrique. En outre, depuis les années quatre-vingt, des pays comme le Pérou et le Mexique, qui ont poursuivi des politiques de substitution des importations et de protection de leurs industries naissantes, ont été totalement surclassés par des pays comme Hong Kong, lequel a toujours opté pour une ouverture complète et une libéralisation de son marché ${ }^{16}$.

Les nouvelles théories de la croissance peuvent-elles faire avancer ce débat? De récents travaux, effectués par Acemoglu-Aghion-Zilibotti (2002) suggèrent qu'elles le peuvent, pourvu qu'elles s'appuient sur une description plus détaillée du processus par lequel les améliorations de productivité se produisent dans des pays (ou des secteurs) situés en deçà de la «frontière technologique ». L'idée de base d'Acemoglu-Aghion-Zilibotti (2002) - dorénavant désigné par AAZ - est que ces améliorations de productivité dans les secteurs en deçà de la frontière technologique, nécessitent à la fois l'imitation de technologies d'avant-garde et l'innovation au-delà des technologies locales préexistantes. Si l'imitation peut

16. Après une période de croissance dont le taux moyen se situait dans les 3,5\%-4 \% entre 1950 et le milieu des années soixante-dix, le Brésil et le Pérou avaient atteint un produit intérieur brut par travailleur correspondant à $35 \%$ de celui des États-Unis. Par la suite, ces deux pays ont plafonné à ce niveau. À l'opposé, nous constatons que des pays comme Hong Kong, dont le produit intérieur brut par travailleur n'arrivait qu'à seulement $17 \%$ de celui des États-Unis en 1960, a surpassé le Brésil et le Pérou pendant les années quatre-vingt et les années quatre-vingt-dix, atteignant $70 \%$ du niveau des États-Unis en 2000 (voir Acemoglu et alii). 
tirer avantage de l'expérience des entreprises en place et des gestionnaires en fonction, des gestionnaires chevronnés peuvent employer les connaissances qu'ils ont acquises sur le tas pour gérer des investissements de plus en plus importants avec le temps; l'innovation exige la sélection des meilleurs managers et la correspondance optimale entre les gestionnaires et les entreprises. (Notez qu'en discutant la convergence dans la section précédente, nous n'avons pas distingué entre ces deux dimensions du processus de diffusion technologique.)

Cette dichotomie entre l'imitation et l'innovation dans le processus de diffusion technologique implique que des politiques (ou des institutions) différentes maximiseront la croissance à des étapes distinctes de développement, par exemple, pour des secteurs ou des pays où la technologique n'en est pas au même point par rapport à la frontière technologique. Ainsi, dans des économies ou des secteurs très en deçà de la technologie de pointe, l'imitation des technologies d'avantgarde maximisera le taux moyen d'amélioration de la productivité, bien qu'une telle imitation puisse être empêchée par des contraintes sur le crédit et l'indisponibilité des fonds; mais, en enlevant ces contraintes, une stratégie basée sur l'investissement qui s'appuierait sur du financement à long terme et sur une protection douanière ou des subventions pourrait alors rehausser la croissance, car l'imitation favorise les relations contractuelles à long terme et l'acquisition de l'expérience. Cependant, dans des économies ou des secteurs qui sont presque rendus à la frontière technologique, la croissance peut être mieux stimulée par une stratégie reposant sur l'innovation et qui s'en remettrait à la concurrence sur le marché pour favoriser les gestionnaires les plus innovateurs.

Jusqu'à maintenant, nous avons raisonné en termes d'institutions maximisant la croissance. Cependant, le même arbitrage entre, d'une part, l'expérience du gestionnaire en matière d'imitation et, d'autre part, la sélection des innovations par le marché explique aussi pourquoi, dans des pays très en deçà de la frontière technologique, l'équilibre est atteint lorsque les gestionnaires/entrepreneurs obtiennent du refinancement indépendamment de leurs réalisations antérieures, tandis que dans les pays qui sont près de la frontière technologique, l'équilibre se réalise lorsque les gestionnaires les moins performants sont éliminés ${ }^{17}$.

\subsection{Institutions endogènes et convergence technologique : un exemple ${ }^{18}$}

L'exemple suivant explore la relation entre la distance d'une économie par rapport à la frontière technologique mondiale et l'incitation à l'intégration verticale des entreprises. En nous basant sur les mêmes idées que celles de AAZ

17. Pourtant, le passage d'une relation contractuelle de court terme à une relation de long terme (ou d'un refinancement non sélectif des gestionnaires/entrepreneurs à un refinancement sélectif) peut se produire trop tard dans le processus de développement technologique d'un pays. Comme AAZ (2002a) l'a démontré, cette situation n'enraie pas seulement la croissance, mais peut aussi empêcher la productivité du pays de converger vers la frontière technologique.

18. Cette sous-section est entièrement tirée d'Acemoglu-Aghion-Zilibotti (2002b). 
(2002a), nous montrerons que l'incitation à l'intégration verticale est d'autant plus forte que l'économie est loin de la frontière technologique. En outre, nous voudrons examiner comment la distance d'un pays de la frontière technologique mondiale a des répercussions sur le type de contrats que l'entreprise signe avec ses gestionnaires, ses bailleurs de fonds et ses fournisseurs et, inversement, comment ces contrats peuvent affecter la croissance économique.

Notre théorie de l'intégration verticale allie Grossman-Hart (1986) avec Aghion-Tirole (1997). Le premier article développe une théorie de l'intégration verticale atténuant les problèmes de hold up entre les entreprises et leurs fournisseurs. En modifiant la propriété de l'actif, et donc les options de désengagement des parties, l'intégration verticale affecte la répartition ex post de la rente, et de là, les motivations à l'investissement des diverses parties. À ce cadre conceptuel, nous ajoutons la question de la surcharge de gestion, analysée dans Aghion et Tirole (1997). Dans notre théorie, les bénéfices de l'intégration verticale proviennent du fait que la société mère n'a pas à partager la rente avec ses fournisseurs, car la plupart de ses activités sont menées en son sein. Le coût de l'intégration verticale, d'autre part, provient de la surcharge de gestion (que l'on peut modéliser avec une fonction convexe de coûts), qui réduit les investissements dans certaines activités, en particulier dans des activités innovatrices. Élaborant sur les mêmes idées que celles de AAZ (2002b), nous soutenons que les activités d'innovation deviennent d'autant plus importantes que l'économie s'approche de la frontière technologique mondiale. Ainsi, il arrive un temps où il devient justifiable d'encourir les coûts du partage de la rente liée à l'impartition de certaines activités de production, pour pouvoir profiter d'innovations plus importantes.

\subsubsection{Le modèle de base}

Le modèle de croissance sous-jacent est identique à celui de la section 2.2, sauf que le temps est discontinu. L'économie est formée d'un continuum de générations d'agents, ne se chevauchant pas et ne durant qu'une période. Chaque génération est formée d'un « capitaliste », qui détient initialement les droits de propriété des « sites de production » et d'un groupe de $1+L$ travailleurs, dont certains peuvent aussi remplir la fonction de gestionnaire. Chaque travailleur est doté d'une unité de travail qu'il fournit d'une façon inélastique et sans désutilité. Tous les individus sont indifférents à l'égard du risque.

Il existe un seul bien final, qui sert aussi de numéraire, et qui est produit dans un contexte concurrentiel en employant du travail et un continuum d'intrants intermédiaires selon l'équation :

$$
y_{t}=\frac{1}{\alpha} L^{1-\alpha}\left[\int_{0}^{1}\left(s_{t}(v) A_{t}(v)\right)^{1-\alpha} x_{t}(v)^{\alpha} d v\right]
$$

où $s_{t}(v) A_{t}(v)$ est la productivité dans le secteur $v$ au moment $t, x_{t}(v)$ dénote le flux d'intrants intermédiaires utilisés dans la production finale, toujours au temps $t$, 
$\alpha \in[0,1] . s_{t}(v)$ correspond à la composante de la productivité découlant des investissements spécifiques de la firme, et $A_{t}(v)$ décrit l'état de la technologie dans ce secteur, déterminé par les activités d'innovation et d'imitation.

Dans chaque secteur intermédiaire $v$, un producteur intermédiaire peut produire une unité de bien intermédiaire avec la technologie de pointe $A_{t}(v)$, en utilisant une unité de bien final comme capital. Les investissements spécifiques de la firme et les améliorations de productivité sont décidés au début de la période. Chaque producteur intermédiaire fait face ex post à la concurrence de divers imitateurs, ce qui le force à prendre le prix limite : $p_{t}(v)=\chi$. Conséquemment, le profit de chaque entreprise du secteur intermédiaire est $\pi_{t}(v)=\left(p_{t}(v)-1\right) x_{t}(v)$. En faisant les substitutions pour le prix limite et le choix maximisant bénéfice de $x_{t}(v)$, nous en arrivons à l'équilibre du profit monopolistique :

$$
\pi_{t}(v)=\delta s_{t}(v) A_{t}(v) L
$$

où $\delta \equiv(\chi-1) \chi^{-\frac{1}{1-\alpha}}$ est une mesure du degré de concurrence dans l'économie et une fonction monotone croissante de $\chi$. Lorsque $\chi$, ou d'une façon équivalente $\delta$, est élevé - éventuellement pour des raisons technologiques ou de réglementation gouvernementale -, la concurrence sera moins vive, et le prix et le profit monopolistique seront plus considérables.

Maintenant définissons :

$$
A_{t} \equiv \int_{0}^{1} A_{t}(v) d v
$$

comme la productivité moyenne dans le pays au moment $t, \bar{A}_{t}$ la productivité de pointe mondiale (l'état de la technologie des différents pays étant $A_{t} \leq \bar{A}_{t}$ ), et

$$
a_{t}=\frac{A_{t}}{\overline{A_{t}}}
$$

une mesure (inverse) de la distance du pays jusqu'à la frontière technologique.

Le principal ajout au modèle de la section 2.2 consiste à spécifier la dynamique de la productivité en fonction des activités d'innovation et de la diffusion des connaissances. Ici, nous supposerons qu'avant que la production n'ait lieu, les entreprises intermédiaires peuvent accroître leur productivité par l'imitation des technologies de pointe ou par l'amélioration novatrice des technologies existantes dans le pays. L'imitation est directe, mais l'innovation exige des investissements et des habiletés de la part des entrepreneurs/gestionnaires, et son succès variera selon les entreprises et selon le temps. Plus précisément, nous supposons :

$$
A_{t}(\mathrm{v})=\eta_{t-1}+x_{t}(\mathrm{v}) A_{t-1}
$$

où

$$
x_{t}(\mathrm{v})=x(v)
$$


est la probabilité qu'une innovation dans le secteur $v$ au temps $t$ soit couronnée de succès. En cas de succès, il y a innovation de quantité 1 . Dans le cas contraire, il n'y a pas d'innovation du tout. Comme l'innovation repose sur les connaissances acquises dans le pays, on la multiplie par $A_{t-1}$. D'autre part, le premier terme dans (16) reflète l'imitation de la technologie mondiale de pointe, d'où le terme en $\bar{A}_{t-1}$.

Concentrons-nous sur un équilibre symétrique dans lequel toutes les entreprises intermédiaires d'un pays choisiront la même intensité d'innovations $x(v)=x$. Alors, en divisant les deux membres de l'équation précédente par $\bar{A}_{t-1}$ et en intégrant par rapport à $v$, nous obtenons une simple équation linéaire, décrivant comment la variable d'état $a_{t}=\frac{A_{t}}{A_{t}}$ évolue avec le temps :

$$
a_{t}=\frac{1}{1+g}\left(n+x a_{t-1}\right)
$$

où $g$ est le taux de croissance de la frontière technologique mondiale ${ }^{19}$.

\subsubsection{La distance jusqu'à la frontière et l'intégration verticale}

Nous analyserons maintenant la décision d'intégration verticale des firmes (du secteur intermédiaire). Ces firmes (ou leurs propriétaires/capitalistes) peuvent choisir une structure verticalement intégrée, selon laquelle elles sont responsables aussi bien des activités d'innovation que de la production. Ou alors, elles peuvent opter pour l'impartition d'une partie de leurs activités de production ou d'innovation. Ici, pour des considérations d'espace, nous nous concentrerons uniquement sur le cas de l'impartition des activités de production. L'idée de base est que l'investissement dans une échelle de production et d'innovation exige des efforts, et peut-être plus important encore, du temps et une échelle de priorités. Dans une structure verticalement intégrée, cela crée une surcharge pour le gestionnaire. L'impartition atténue cette surcharge certes, mais elle occasionne un problème de hold $u p^{20}$ : un nouvel agent fait son apparition, qui partagera une partie de la rente avec le propriétaire, réduisant ainsi le gain de ce dernier, et conséquemment, son incitation à l'investissement.

19. Que les investissements spécifiques de la firme, $s$, ne contribuent pas au progrès technologique est sans conséquence pour ce résultat.

20 À l'instar de Grossman et Hart (1986) et des travaux sur les contrats incomplets, nous supposons qu'aucun contrat ex ante ne peut prévoir un partage exécutoire du surplus; la répartition des profits ne peut donc se faire que par une négociation ex post. De plus, nous ne considérons pas ici le cas où le propriétaire ne recourt pas à l'impartition, mais embauche plutôt le fournisseur. Dans la mesure où cet employé réalise des investissements importants pour l'entreprise, il disposera vraisemblablement d'un pouvoir de négociation ex post, tout en étant sujet au contrôle du propriétaire. Ainsi, lorsque le fournisseur devient un employé, l'organisation est d'un type intermédiaire, se situant entre la structure verticalement intégrée et l'impartition. Nous remettons à plus tard la comparaison entre l'impartition et l'intégration verticale avec ou sans embauche d'agents supplémentaires. 
Nous modélisons la surcharge de gestion en ayant recours à une fonction convexe du coût de l'effort; plus l'effort que le gestionnaire déploie dans l'intérêt du propriétaire est grand, plus le coût marginal d'un nouvel effort est élevé. Pour formaliser cela, considérons la fonction de coûts suivante dans le cas d'une entreprise verticalement intégrée :

$$
C_{t}(s, x)=\left\{\begin{array}{l}
k \bar{A}_{t-1} \quad \text { si } s+x \leq T \\
\infty \quad \text { autrement }
\end{array} .\right.
$$

Cette fonction de coûts spécifie qu'il existe un coût fixe constant $k \bar{A}_{t-1}$ (qui s'accroît en fonction de l'état de la technologie mondiale) et que le coût marginal de l'effort reste à 0 jusqu'à ce que $s+x$ atteigne $T$, avant de devenir infini par la suite (ainsi, nous avons une courbe de coût marginale en forme de $L$ - extrêmement convexe). La motivation intuitive d'une telle fonction est que le gestionnaire (propriétaire) dispose d'un laps de temps total $T$, que l'expansion de la capacité productive nécessite une unité de temps et que les activités d'innovation prennent aussi une unité de temps. Lorsque ces activités auront accaparé tout le temps disponible, le gestionnaire ne pourra plus accroître son effort.

Nous supposons que

$\eta \geq T$.

Cette hypothèse garantit la « rareté » du temps pour le propriétaire, de telle sorte que l'arbitrage évoqué est plus intéressant.

Pour les propriétaires d'une entreprise intégrée, le problème de maximisation se pose directement comme suit : choisir $(s, x)$ de façon à maximiser

$$
\pi_{t}(v)=\delta s_{t}(v) A_{t}(v) L-C_{t}(s, x)=\delta L s(\eta+x a) \bar{A}_{t-1}-C_{t}(s, x)
$$

où la deuxième égalité repose sur le fait que toutes les entreprises choisiront les mêmes $x$ et $s$, et après avoir fait la substitution de $A_{t}(v)$ avec les équations (15) et (16).

L'hypothèse (A1), c'est-à-dire, la pénurie de temps des gestionnaires, implique directement que l'optimum $x$ doit être égal à zéro ${ }^{21}$.

Autrement dit, dans une entreprise verticalement intégrée, la surcharge d'effort décourage l'innovation. L'échelle optimale de production correspond alors simplement à : $s=T$. Cela donne ainsi un niveau d'équilibre pour l'utilité du propriétaire égal à :

21. La condition de premier ordre pour une solution intérieure est :

$$
s=\frac{\eta+\underline{\gamma} a+T a}{2 a} .
$$

Une condition suffisante pour $s \geq T$, et donc pour $x=0$, est $\eta+\gamma \geq T$, ce qui est garanti par l'hypothèse (A1). 


$$
U_{t}^{V I}=u^{V I}(\mathrm{a}) \bar{A}_{t-1}=(\delta L T \eta \gamma a-k) \bar{A}_{t-1} .
$$

Maintenant, tournons-nous vers le cas où les activités de production sont imparties à un fournisseur indépendant (nous l'appellerons le cas de la non-intégration). La fonction de coûts du fournisseur est

$$
C^{s}(s)=\left\{\begin{array}{ll}
\frac{1}{2} k \bar{A}_{t-1} \quad \text { si } s \leq T \\
\infty & \text { autrement }
\end{array} .\right.
$$

Autrement dit, le fournisseur dispose aussi d'un temps total $T$, qu'il alloue simplement aux activités productives. Il encourt alors le coût fixe $\frac{1}{2} k \bar{A}_{t-1}$, qui est une fraction du coût fixe de production supporté par la société verticalement intégrée. Lorsque ces activités de production sont imparties, la fonction de coûts du propriétaire est :

$$
C_{t}^{x}(x)=\left\{\begin{array}{ll}
\frac{1}{2} k \bar{A}_{t-1} \quad \text { si } x \leq T \\
\infty & \text { autrement }
\end{array} .\right.
$$

Donc, le propriétaire peut maintenant allouer tout son temps aux activités d'innovation, et il encourt le coût fixe de production $\frac{1}{2} k \bar{A}_{t-1}$. Nous supposons aussi que les fournisseurs peuvent toujours travailler comme des salariés, de telle sorte que même dans le cas comportant impartition, la main-d'oeuvre totale travaillant dans le secteur intermédiaire est égale à $L$.

À l'instar de Grossman et Hart (1986) et des travaux sur les contrats incomplets, nous supposons que les décisions d'échelle et de productivité ne sont pas vérifiables ex post par des tiers et ne peuvent donc pas faire l'objet d'un contrat ex ante. Ainsi le propriétaire et le fournisseur doivent compter sur des négociations ex post pour déterminer comment les profits seront partagés entre eux. Cela crée un problème de hold up classique : une partie de la rente doit être partagée ex post avec le fournisseur. Pour simplifier la discussion, nous supposons que le propriétaire et le fournisseur disposent d'une option de désengagement égale à zéro (c'est-à-dire, une fois que des activités productives sont imparties et que les décisions d'investissement sont prises, le propriétaire ne peut plus effectuer d'activités productives en cas de désaccord). En supposant que le propriétaire et le fournisseur disposent du même pouvoir de négociation concernant le surplus ex post, chaque partie obtient $\frac{1}{2}$ du surplus ex post, à savoir ${ }^{22}$ :

22. Nous supposons que ce surplus est assez considérable pour couvrir les frais fixes du fournisseur. 


$$
\frac{1}{2} \delta L s(\eta+x a) \bar{A}_{t-1}
$$

Alors, pour une échelle donnée $s$, la firme choisira son intensité d'innovation $x$ pour :

$$
\max _{x} \frac{1}{2} \delta L s(\eta+x a) \bar{A}_{t-1}-C^{x}(x)
$$

l'intensité d'innovation $x$ lui étant donnée, le fournisseur investira dans une échelle de production de façon à :

$$
\max _{s} \frac{1}{2} \delta L s(\eta+x a) \bar{A}_{t-1}-C^{s}(s)
$$

Les investissements d'équilibre dans l'échelle de production et l'innovation seront tout simplement égaux à :

$$
s^{N I}(a)=T \quad \text { et } \quad x^{N I}(a)=T .
$$

Remarquez que dans ce cas, la nature non coopérative du rapport entre le fournisseur et le propriétaire ne crée pas de sous-investissement, puisque le coût marginal de l'investissement est 0 . Dans un modèle plus général, le sous-investissement se produirait et découragerait aussi l'impartition. À partir de (20), l'utilité d'équilibre ex ante pour la firme est égale à :

$$
U_{t}^{N I}=u^{N I}(a) \bar{A}_{t-1}=\left(\frac{1}{2} \delta L T(\eta+T a)-\frac{1}{2} k\right) \bar{A}_{t-1}
$$

L'hypothèse (A1) implique directement que :

$$
\frac{d u^{N I}}{d a}>\frac{d u^{V I}}{d a}
$$

Autrement dit, plus grand est $a$, c'est-à-dire plus le pays se trouve près de la frontière technologique, plus la valeur de l'innovation est élevée et donc plus grande est l'utilité de l'impartition. Intuitivement, la non-intégration (l'impartition) devient d'autant plus coûteuse pour le propriétaire que $a$ augmente, puisqu'un $a$ plus élevé implique une production accrue, qui sera finalement partagée avec le fournisseur. D'autre part, avec l'augmentation de $a$, l'innovation devient plus importante, et la valeur de l'impartition s'accroît. L'hypothèse (A1) garantit que le deuxième effet dominera le premier.

Nous déterminerons maintenant la décision d'intégration d'équilibre, en fonction de la distance du pays jusqu'à la frontière technologique (mesurée par $a$ ). Les propriétaires (capitalistes) doivent décider si la firme restera intégrée ou s'ils 
auront recours à l'impartition des activités de production. Donc, pour déterminer la forme organisationnelle d'équilibre, nous devons comparer $u^{V I}(a)$ donné par (19) à $u^{N I}(a)$ donné par $(21)^{23}$.

Premièrement, supposons que $a=0$, alors :

$$
u^{N I}(0)=\frac{\delta L T}{2} \eta-\frac{1}{2} k<u^{V I}(0)=\delta L T \eta-k
$$

(n'oubliez pas que $\varphi \geq \frac{1}{2}$. Ainsi dans des économies loin de la frontière technologique mondiale, les propriétaires préfèrent rester verticalement intégrés : l'innovation a relativement peu d'importance en regard de l'imitation, donc l'impartition a peu d'avantages et les coûts privés sont élevés à cause du problème de hold up. Ce résultat reste valable malgré le fait que d'un strict point de vue technologique, l'impartition est supérieure à l'intégration verticale : le fournisseur n'a aucun coût d'opportunité (comme il travaille toujours dans la production), et les investissements dans l'innovation peuvent être plus considérables. Néanmoins, du point de vue du propriétaire, l'impartition entraîne un coût privé, puisque le fournisseur touche une partie de la rente.

En posant $u^{V I}\left(a^{v}\right)=u^{N I}\left(a^{v}\right)$ d'après (19) et (21), nous en arrivons au seuil critique

$$
a^{v} \equiv \frac{n-\frac{1}{2} k / \delta L}{T} .
$$

Pour tout $a<a^{v}$, la forme organisationnelle d'équilibre sera l'intégration verticale et pour $a>a^{v}$, ce sera l'impartition. Le seuil $a^{v}$ est généralement inférieur à 1 , de telle sorte qu'il y aura une substitution en faveur de l'impartition avant d'atteindre la frontière technologique. Remarquez qu'un $a^{v}$ inférieur à 1 est d'autant plus probable lorsque le coût de production fixe $k$ est élevé, que la concurrence est vive (c'est-à-dire que $\delta$ est petit), ou que $\eta$ est faible. Plus généralement, on peut montrer facilement que $\frac{\partial a^{v}}{\partial \delta}>0$ et $\frac{\partial a^{v}}{\partial \eta} \geq 0$, de telle sorte que l'intégration verticale est plus probable dans des environnements moins concurrentiels et dans des secteurs où l'imitation est relativement importante. Une faible de concurrence crée des rentes élevées, augmentant le coût de hold up sur la partie des activités qui est impartie. Aussi, quand l'imitation est plus importante, les avantages de l'impartition en terme d'innovations sont moins prononcés en regard du coût du partage de la rente.

23. Parce que les activités de production sont imparties à des fournisseurs qui ne possèdent aucune richesse, des contrats stipulant des versements à l'avance de la part des fournisseurs en faveur des propriétaires ne sont pas possibles. Des contrats selon lesquels des fournisseurs potentiels promettent de faire des paiements ex post sont exclus par notre hypothèse sur les contrats incomplets. 


\section{CONCLUSION}

Dans cet exposé, nous avons soutenu que le paradigme schumpetérien de la croissance, selon lequel la croissance résulte d'une suite d'innovations améliorant la qualité - elles-mêmes, fruit de l'action d'entrepreneurs motivés par le profit peut grandement nous aider à relever les trois principaux défis dans l'élaboration d'une bonne théorie de la croissance, à savoir : (i) expliquer les différences d'un pays à l'autre dans les taux de croissance à long terme et déduire des relations vérifiables entre la croissance à long terme et un certain nombre de politiques ou de variables institutionnelles; (ii) comprendre les évolutions historiques, en particulier pourquoi certains pays semblent converger vers le niveau de productivité des pays les plus avancés alors que d'autres pays n'arrivent pas à décoller; (iii) éclairer à la fois les transformations institutionnelles et le changement technologique.

De toute évidence, il reste beaucoup à faire pour relever complètement ces défis. Des nouveaux modèles de la croissance, découlent un certain nombre de prédictions sur les déterminants de la croissance à long terme; mais certaines de ces prédictions se sont avérées contredites par les faits, remettant ainsi en question la validité des théories et soulignant la nécessité de les améliorer. En voici un exemple éloquent : il s'agit de la relation entre la croissance et la concurrence dans une économie de marché. Les économistes se sont intéressés depuis longtemps au rapport entre l'innovation et la concurrence dans une telle économie. Tant les travaux théoriques sur les intrants-extrants que les plus récents qui ont porté sur la croissance endogène abordent la question. Les formulations intrantsextrants standard ${ }^{24}$ prédisent que l'innovation baissera en fonction de la concurrence, car davantage de concurrence réduit les rentes de monopole récompensant les innovateurs qui remportent du succès. Cependant, des travaux empiriques, comme ceux de Nickell (1996) et Blundell et alii (1995), ont trouvé une corrélation positive entre la concurrence sur le marché et l'occurrence des innovations. Pour tenter de réconcilier le paradigme schumpetérien avec les faits révélés par de telles recherches, plusieurs tentatives théoriques ont été faites, produisant des prédictions diverses quant à la forme de la relation entre l'innovation et la concurrence sur le marché 25 .

La tentative la plus récente s'inspire, d'une part, du travail théorique d'Aghion-Harris-Vickers (1997) et d'Aghion-Harris-Howitt-Vickers (2001) qui élargit le modèle schumpetérien de base en permettant aux firmes en place d'innover, et, d'autre part, de la recherche empirique d'Aghion-Bloom-BlundellGriffith-Howitt (2002), cherchant à tester les principales prédictions du modèle remanié. L'ensemble de cette recherche suggère que les nouvelles théories de la croissance et la microéconomie peuvent s'enrichir mutuellement, et, en particulier, qu'un dialogue avec cette dernière peut aider les premières à s'améliorer, notamment pour formuler des réponses plus précises et plus appropriées concernant les déterminants du développement économique.

24. Voir, entre autres, Dasgupta-Stiglitz (1980) et aussi la première génération de modèles de croissance schumpetériens (Aghion-Howitt, 1992; Caballero-Jaffe, 1993).

25. Voir Aghion-Howitt (1998), chapitre 7, pour un aperçu de certaines de ces tentatives. 


\section{BIBLIOGRAPHIE}

Acemoglu, Daron, Aghion, Philippe et Fabrizio Zilibotti (2002a), « Distance to Frontier, Selection, and Economic Growth », NBER Working Paper 9066.

Acemoglu, Daron, Aghion, Philippe et Fabrizio Zilibotti (2002b), « Vertical Integration and Distance to Frontier », mimeo MIT-UCL.

Aghion, Philippe, Nicholas Bloom, Richard Blundell, Rachel Griffith et Peter HowitT (2002), « Competition and Innovation: An Inverted-U Relationship », mimeo UCL.

Aghion, Philippe, Christopher Harris et John Vickers (1997), « Competition and Growth with Step-by-Step Innovation: An Example », European Economic Review, Papers and Proceedings : 771-782.

Aghion, Philippe, Christopher Harris, Peter Howitt et John Vickers (2001), «Competition, Imitation and Growth with Step-by-Step Innovation. », Review of Economic Studies.

Aghion, Philippe et Peter Howitt (1992), « A Model of Growth Through Creative Destruction. », Econometrica, 60 : 323-51.

Aghion, Philippe et Peter Howitt (1998), Endogenous Growth Theory, Cambridge : MIT Press.

Aghion, Philippe et Jean Tirole (1997), « Formal and Real Authority in Organizations », Journal of Political Economy, 105 : 1-29.

Barro, Robert et Xavier Sala-I-Martín (1995), Economic Growth, New York : McGraw-Hill.

Blundell, Richard, Rachel Griffith et John Van Reenen (1995), « Dynamic Count Data Models of Technological Innovation », Economic Journal, 105 : 333-344.

Caballero, Ricardo et Adam Jaffe (1993), « How High are the Giants' Shoulders? An Empirical Assesment of Knowledge Spillovers and Creative Destruction in a Model of Economic Growth », NBER Macroeconomic Annual : $15-74$.

Dasgupta, Farthe et Joseph Stiglitz (1980), « Industrial Structure and the Nature of Innovative Activity », Economic Journal, 90 : 266-293.

De la Fuente, Angel (1995), « Catch-up, Growth and Convergence in the OECD », CEPR Discussion Paper, $\mathrm{n}^{\circ} 1274$.

Frankel, Marvin (1962), «Obsolescence and Technological Change in a Mature Economy », American Economic Review, 55(3) : 296-319.

Gerschenkron, Alexander (1962), Economic Backwardness in Historical Perspective, Harvard University Press, Cambridge MA.

Grossman, Sanford et Oliver Hart (1986), « The Costs and Benefits of Ownership: A Theory of Vertical and Lateral Integration », Journal of Political Economy, 94 : 691-719.

Grossman, Gene et Ethanan Helpman (1991), Innovation and Growth in the Global Economy, Cambridge, MA : MIT Press. 
Hausmann, Ricardo et Ani Rodrik (2002), « Economic Development As SelfDiscovery », mimeo Harvard University.

Helliwell, John F. et Alan Chung (1991), « Macroeconomic Convergence: International Transmission of Growth and Technical Progress. » in PETER HOOPER et J. David Richardson (éds), International Economic Transactions: Issues in Measurement and Empirical Research. NBER Studies in Income and Wealth, Vol. 55, Chicago : The University of Chicago Press, p. 388-436.

Howitt, Peter (2000), « Endogenous Growth and Cross-Country Income Differences », American Economic Review, 90 : 829-846.

Howitt, Peter et David Mayer-Faulkes (2002), « R+D, Implementation and Stagnation: A Schumpeterian Theory of Convergence Cluks », NBER Working Paper 9104.

Jones, Larry, Rodolfe Manuelli et Ennio Stacchetti (1999), « Technology (and Policy) Shocks in Models of Endogerous Growth » Working Paper.

Kortum, Samuel (1997), « Research, Patenting and Technological Changes », Econometrica, 65(6) : 1 389-1 419.

Lucas, Robert E. (1988), « On the Mechanics of Economic Development », Journal of Monetary Economics, 22(1) : 3-42.

Malinvaud, Edmond (1982), Théorie macroéconomique, Dunod.

Mankiw, N. Gregory, David Romer et David N. Weil (1992), « A Contribution to the Empirics of Economic Growth », Quarterly Journal of Economics, 107(2) : 407-437.

Nickell, Stephen (1996), « Competition and Corporate Performance », Journal of Political Economy, 104 : 724-46.

Romer, PAul M. (1986), «Increasing returns and Long Run Growth », Journal of Political Economy, 94 (5) : 1 002-1 037.

Romer, Paul M. (1990), «Endogenous Technological Change », Journal of Political Economy, 98(5) : 71-102.

Segerstrom, Paul, T. Anant et Elias Dinopoulos (1990), « A Schumpeterian Model of the Product Life Cycle », American Economic Review, 80 : 1 0771092.

Shleifer, Andrei et Robert Vishny (1999), The Grabbing Hand, Harvard University Press.

Solow, Robert M. (1956), «A Contribution to the Theory of Economic Growth », Quarterly Journal of Economics : 65-94.

Stiglitz, JosePh (1995), Wither Socialism, MIT Press.

Swan, T.W. (1956), « Economic Growth and Capital Accumulation », Economic Record, 32 : 34-361. 